\section{Fat and skinny bacteria?}

Abby L. Geis

\section{INTRODUCTION}

"Correspondence to: Abby L. Geis, Arkansas College of Osteopathic Medicine, Fort Smith, AR 72916 abby.geis@arcomedu.org

Cite This Article:

Geis, A.L. 2018. Fat and skinny bacteria?. Diabesity 4(2): 5-8. DOI: 10.15562/ diabesity.2018.47
Due to the increased availability of high-throughput DNA-sequencing technologies and quantitative PCR, investigators have flooded the scientific literature with reports describing the composition of different gastrointestinal microbiotas. With the use of gnotobiotic isolators housing axenic animals, i.e. germ- or microbiota-free animals, investigators have also undertaken more hypothesis-driven research addressing putative roles for the microbiota in host health. Possibly inspired by the patent spread and pathogenesis of obesity and metabolic syndrome, in combination with the observation that a commonly used strain of germ-free laboratory mice are more resistant to obesity than their conventional counterparts, ${ }^{1}$ a major focus of much of this work has considered the relationship between the microbiota of the host and the susceptibility of the host to obesity. Additionally, compared to many existing and theoretic therapeutics to counter the obesity pandemic, the microbiota is potentially a more practical target. I attempt to very briefly summarize, for nonprofessionals of microbiome study, the composition of the microbiota as it relates to obesity in humans. This is intended to provide the reader with sources for finding further information, including primary research that has contributed to our knowledge and more comprehensive reviews on the relevant topics mentioned.

\section{COMPOSITION OF THE OBESE MICROBIOTA}

\section{Rejecting the Bacteroidetes: Firmicutes ratio}

Bacterial 16S DNA sequencing of stool samples from obese and non-obese individuals has revealed significant differences in their respective microbiotas. ${ }^{2-7}$ Indeed, the microbiota isolated from obese individuals is commonly designated as "dysbiotic," a term meaning microbial imbalance or abnormal microbiota. However, how the microbiota is abnormal is not at all well understood, largely because what makes a healthy microbiota is not well understood. A frequently reported measure describing the microbiota is the ratio of Bacteroidetes to Firmicutes, two phyla that represent the most abundant bacterial species in human stool. ${ }^{8,9}$ More often, a decreased ratio-where Bacteroidetes are under-represented and Firmicutes are over-represented-is reported in obesity compared to healthy-weight controls, ${ }^{6,10-12}$ yet some reports have identified an increased ratio of Bacteroidetes to Firmicutes. ${ }^{5,13}$ Still others have reported an increase in the abundance of both phyla in the microbiota of obese individuals, ${ }^{14}$ while additional studies have found no association between the abundance of Bacteroidetes nor Firmicutes in the microbiotas of obese and healthy-weight individuals. ${ }^{4,9,15}$ Thus, the actual significance of the relative abundance of the two phyla, which are composed of thousands of distinct bacterial species, is extremely controversial. For a more comprehensive review of the members of Bacteroidetes and their connections to obesity, see Johnson et al. ${ }^{16}$

\section{Diversity is not created equally}

Most commonly, and perhaps less controversially, the microbiota of obese individuals is described as having reduced bacterial diversity. Of course, how diversity is calculated and reported is not entirely consistent, but certain trends have emerged, particularly the obese microbiota exhibiting reduced richness, a measure of a-diversity (within a single sample) which signifies the number of unique microbial species. ${ }^{4,7}$ For instance, in a fairly large study of nearly 600 American adults, Peters et al observed that the richness of the microbiota of obese individuals was significantly reduced compared to that of overweight (but not obese) and healthyweight participants. However, when accounting for the evenness of the microbial populations within each sample, differences in $\alpha$-diversity failed to reach significance. ${ }^{4}$ Another fairly large study of approximately 300 Danish individuals found that the sampled microbiotas demonstrated a bimodal distribution with regard to richness. ${ }^{7}$ Significantly more individuals in the group with lower microbial richness were obese and showed increased markers of metabolic syndrome, e.g. increased serum leptin, triglycerides, and hyperinsulinemia. Furthermore, in both this and an accompanying study, ${ }^{17}$ microbial genes for metabolic pathways associated with inflammatory stress and production of potentially 
deleterious metabolites were proportionally increased in the microbiomes with decreased richness. Interestingly though, when comparing the microbiomes of obese and non-obese individuals, fewer differences were identified than when the groups were divided based on microbial richness. ${ }^{7}$

Other metrics that can be incorporated into measures of diversity, arguably more valuable than richness or evenness alone, include microbial phylogeny ${ }^{18}$ and function. ${ }^{19}$ Phylogenetic diversity takes into account the degree of evolutionary relatedness of the resident microbiota. Several studies already mentioned account for microbial phylogeny when determining the diversity of the population. ${ }^{3,6-9,20}$ Importantly, increased diversity, as measured by $16 \mathrm{~S}$ DNA, does not simply signify a "healthy microbiota". Functional diversity, on the other hand, considers the entire microbial genome, the microbial transcriptome, proteome and/or other microbial-derived metabolites, i.e. the functional collective of the entire microbiome. Significant findings concerning the basic behavioral interactions between members of the microbiota, e.g. reinforcing microbiota stability through microbial interactions, are based on transcriptome or metabolome profiles. ${ }^{21-23}$ It is fairly intuitive to appreciate how increased functional diversity may represent a healthier gut ecosystem. For example, functional diversity and redundancy may enable better adaptation to changes in physiology, ${ }^{24,25}$ diet, ${ }^{9,26}$ or exposures to medication. ${ }^{27}$ Indeed, even though a small study of 25-32 year old twin pairs and their mothers revealed the gut microbiotas of obese individuals were more similar to each other than the similarity of microbiotas of lean participants to each other, no apparent overlap of phylotypes amongst microbial samples emerged. Instead, distinct functional cores, representing key metabolic pathways, emerged between the microbiomes of obese individuals compared to non-obese individuals. ${ }^{3}$

\section{"Specie-fic" differences that are real and reproducible?}

So far the distinction between the microbiota compositions of the obese compared to the non-obese is a matter of reduced number, evenness, relatedness, and/or function of the resident species. Summarizing the relevance of specific microbial differences is complicated by the finding that microbial community variations between studies tend to be much greater than between obese and non-obese groups within studies. ${ }^{28,29}$ In addition, sampling biases and insufficient sequencing depths impact how well microbial samples accurately represent their true ecosystems, and rarefaction performed in order to make comparisons across studies further impacts the accuracy of sampled data. One potentially more conserved gut microbial phenotype correlated with individuals who are obese or have low microbial richness is increased abundance of the less dominant phylum Proteobacteria. $4,7,20$ Although not a specific observation, members of several comprising genera, e.g. Escherichia and Salmonella, represent potential human pathogens..$^{30}$ This is potentially relevant because obesity is also associated with increased inflammation; thus, many have investigated the role of the microbiota in obesity-related inflammation (reviewed by McPhee and Schertzer). ${ }^{31}$ Alternatively, Dao et al found that increased or high abundance of Akkermansia muciniphila (in addition to overall microbial richness) in overweight and obese subjects after six weeks on a calorie restricted diet was associated with improved metabolic profiles. ${ }^{32}$ Importantly, $A$. muciniphila has been identified by several investigators as a species capable of conferring protection against obesity and metabolic disorders in animals, ${ }^{33-36}$ so the case for an effective probiotic is arguably best-made in support of A. muciniphila. ${ }^{37}$ Despite the paucity of more specific, reproducible microbial associations with the obese phenotype, some studies have demonstrated that obese and lean human fecal microbiotas can transfer those phenotypes to animals, ${ }^{38-40}$ demonstrating that perhaps a variety of microbiota compositions may impact susceptibility to obesity. Additional research with animal models has provided significantly more insight into the composition and role of the gut microbiota in obesity and metabolic syndrome, as well as revealed possible mechanisms supporting their relationship, ${ }^{1,6,41-47}$ but how well the results from animal studies reflect the gut microbiota and obesity in humans is still unknown.

\section{CONCLUSION}

In spite of the large amount of available data describing microbial associations with human obesity, microbial-related mechanisms that impact host metabolism, and the transmissibility of obesity, especially in animal-to-animal models, there are no clear obesogenic microbiota phenotypes. Indeed, few compositional characteristics of the obese microbiotas considered herein appeared to be conserved across different studies, with the possible exception of some measure of reduced diversity compared to the non-obese microbiotas. It seems as though future work may unravel distinct, organism-independent, microbial functions that, when combined with certain dietary inputs or physiological aspects of the host, may enhance 
susceptibility to obesity. Beyond the scope of this commentary, but critical to the understanding of a putatively obesogenic microbiota, is the irrefutable role of diet on the composition and metabolism of the microbiota, as well as on the metabolism of the host (reviewed by Sonnenburg and Backhed). ${ }^{48}$ Naturally, what we feed ourselves directly impacts what we feed our microbiota, and the functional metabolic pathways expressed by the resident microbiota will influence which organisms can flourish with those dietary inputs and what microbiota-derived metabolites are produced. Future work describing the compositional and functional variation that is possible across different healthy microbiotas, as well as compositional and/or functional similarities conserved across different healthy microbiotas, should provide a better platform for investigating, and eventually understanding, the abnormal character of a "dysbiotic" microbiota, as it relates to any human pathology.

\section{REFERENCES}

1. Backhed F, Ding H, Wang T, et al. The gut microbiota as an environmental factor that regulates fat storage. Proc Natl Acad Sci U S A. 2004;101(44):15718-15723.

2. Million M, Maraninchi M, Henry M, et al. Obesityassociated gut microbiota is enriched in lactobacillus reuteri and depleted in bifidobacterium animalis and methanobrevibacter smithii. Int J Obes (Lond). 2012;36(6):817825. doi: 10.1038/ijo.2011.153 [doi].

3. Turnbaugh PJ, Hamady M, Yatsunenko T, et al. A core gut microbiome in obese and lean twins. Nature. 2009;457(7228):480-484. doi: 10.1038/nature07540 [doi].

4. Peters BA, Shapiro JA, Church TR, et al. A taxonomic signature of obesity in a large study of american adults. Sci Rep. 2018;8(1):9749-018-28126-1. doi: 10.1038/s41598018-28126-1 [doi].

5. Schwiertz A, Taras D, Schafer K, et al. Microbiota and SCFA in lean and overweight healthy subjects. Obesity (Silver Spring). 2010;18(1):190-195. doi: 10.1038/oby.2009.167 [doi].

6. Ley RE, Turnbaugh PJ, Klein S, Gordon JI. Microbial ecology: Human gut microbes associated with obesity. Nature. 2006;444(7122):1022-1023. doi: 4441022a [pii].

7. Le Chatelier E, Nielsen T, Qin J, et al. Richness of human gut microbiome correlates with metabolic markers. Nature. 2013;500(7464):541-546. doi: 10.1038/nature12506 [doi].

8. Lloyd-Price J, Mahurkar A, Rahnavard G, et al. Strains, functions and dynamics in the expanded human microbiome project. Nature. 2017;550(7674):61-66. doi: 10.1038/nature23889 [doi].

9. Jumpertz R, Le DS, Turnbaugh PJ, et al. Energy-balance studies reveal associations between gut microbes, caloric load, and nutrient absorption in humans. Am J Clin Nutr. 2011;94(1):58-65. doi: 10.3945/ajcn.110.010132 [doi].

10. Koliada A, Syzenko G, Moseiko V, et al. Association between body mass index and firmicutes/bacteroidetes ratio in an adult ukrainian population. BMC Microbiol. 2017;17(1):120-017-1027-1. doi: 10.1186/s12866-0171027-1 [doi].

11. Kasai C, Sugimoto K, Moritani I, et al. Comparison of the gut microbiota composition between obese and nonobese individuals in a japanese population, as analyzed by terminal restriction fragment length polymorphism and next-generation sequencing. BMC Gastroenterol. 2015;15:100-015-0330-2. doi: 10.1186/s12876-015-0330-2 [doi].
12. Bervoets L, Van Hoorenbeeck K, Kortleven I, et al. Differences in gut microbiota composition between obese and lean children: A cross-sectional study. Gut Pathog. 2013;5(1):10-4749-5-10. doi: 10.1186/1757-4749-5-10 [doi].

13. Duncan SH, Lobley GE, Holtrop G, et al. Human colonic microbiota associated with diet, obesity and weight loss. Int J Obes (Lond). 2008;32(11):1720-1724. doi: 10.1038/ ijo. 2008.155 [doi].

14. Abdallah Ismail N, Ragab SH, Abd Elbaky A, Shoeib AR, Alhosary Y, Fekry D. Frequency of firmicutes and bacteroidetes in gut microbiota in obese and normal weight egyptian children and adults. Arch Med Sci. 2011;7(3):501-507. doi: 10.5114/aoms.2011.23418 [doi].

15. Arumugam M, Raes J, Pelletier E, et al. Enterotypes of the human gut microbiome. Nature. 2011;473(7346):174-180. doi: 10.1038/nature09944 [doi].

16. Johnson EL, Heaver SL, Walters WA, Ley RE. Microbiome and metabolic disease: Revisiting the bacterial phylum bacteroidetes. J Mol Med (Berl). 2017;95(1):1-8. doi: 10.1007/s00109-016-1492-2 [doi].

17. Cotillard A, Kennedy SP, Kong LC, et al. Dietary intervention impact on gut microbial gene richness. Nature. 2013;500(7464):585-588. doi: 10.1038/nature12480 [doi].

18. McCoy CO, Matsen FA,4th. Abundance-weighted phylogenetic diversity measures distinguish microbial community states and are robust to sampling depth. PeerJ. 2013;1:e157. doi: 10.7717/peerj.157 [doi].

19. Flynn DF, Mirotchnick N, Jain M, Palmer MI, Naeem S. Functional and phylogenetic diversity as predictors of biodiversity--ecosystem-function relationships. Ecology. 2011;92(8):1573-1581.

20. Verdam FJ, Fuentes S, de Jonge C, et al. Human intestinal microbiota composition is associated with local and systemic inflammation in obesity. Obesity (Silver Spring). 2013;21(12):E607-15. doi: 10.1002/oby.20466 [doi].

21. Coyte KZ, Schluter J, Foster KR. The ecology of the microbiome: Networks, competition, and stability. Science. 2015;350(6261):663-666. doi: 10.1126/science.aad2602 [doi].

22. Shoaie S, Karlsson F, Mardinoglu A, Nookaew I, Bordel S, Nielsen J. Understanding the interactions between bacteria in the human gut through metabolic modeling. Sci Rep. 2013;3:2532. doi: 10.1038/srep02532 [doi].

23. Lozupone C, Faust K, Raes J, et al. Identifying genomic and metabolic features that can underlie early successional and opportunistic lifestyles of human gut symbionts. Genome Res. 2012;22(10):1974-1984. doi: 10.1101/gr.138198.112 [doi].

24. Kong LC, Tap J, Aron-Wisnewsky J, et al. Gut microbiota after gastric bypass in human obesity: Increased richness and associations of bacterial genera with adipose tissue genes. Am J Clin Nutr. 2013;98(1):16-24. doi: 10.3945/ ajcn.113.058743 [doi].

25. Palleja A, Kashani A, Allin KH, et al. Roux-en-Y gastric bypass surgery of morbidly obese patients induces swift and persistent changes of the individual gut microbiota. Genome Med. 2016;8(1):67-016-0312-1. doi: 10.1186/ s13073-016-0312-1 [doi].

26. Santacruz A, Marcos A, Warnberg J, et al. Interplay between weight loss and gut microbiota composition in overweight adolescents. Obesity (Silver Spring). 2009;17(10):19061915. doi: 10.1038/oby.2009.112 [doi].

27. Reijnders D, Goossens GH, Hermes GD, et al. Effects of gut microbiota manipulation by antibiotics on host metabolism in obese humans: A randomized double-blind placebo-controlled trial. Cell Metab. 2016;24(1):63-74. doi: 10.1016/j.cmet.2016.06.016 [doi].

28. Finucane MM, Sharpton TJ, Laurent TJ, Pollard KS. A taxonomic signature of obesity in the microbiome? getting to the guts of the matter. PLoS One. 2014;9(1):e84689. doi: 10.1371/journal.pone.0084689 [doi].

29. Walters WA, Xu Z, Knight R. Meta-analyses of human gut microbes associated with obesity and IBD. FEBS Lett. 2014;588(22):4223-4233. doi: 10.1016/j.febslet.2014.09.039 [doi]. 
30. Rizzatti G, Lopetuso LR, Gibiino G, Binda C, Gasbarrini A. Proteobacteria: A common factor in human diseases. Biomed Res Int. 2017;2017:9351507. doi: $10.1155 / 2017 / 9351507$ [doi].

31. McPhee JB, Schertzer JD. Immunometabolism of obesity and diabetes: Microbiota link compartmentalized immunity in the gut to metabolic tissue inflammation. Clin Sci (Lond). 2015;129(12):1083-1096. doi: 10.1042/ CS20150431 [doi].

32. Dao MC, Everard A, Aron-Wisnewsky J, et al. Akkermansia muciniphila and improved metabolic health during a dietary intervention in obesity: Relationship with gut microbiome richness and ecology. Gut. 2016;65(3):426436. doi: 10.1136/gutjnl-2014-308778 [doi].

33. Yang J, Bindels LB, Segura Munoz RR, et al. Disparate metabolic responses in mice fed a high-fat diet supplemented with maize-derived non-digestible feruloylated oligo- and polysaccharides are linked to changes in the gut microbiota. PLoS One. 2016;11(1):e0146144.

34. Zhao S, Liu W, Wang J, et al. Akkermansia muciniphila improves metabolic profiles by reducing inflammation in chow diet-fed mice. J Mol Endocrinol. 2017;58(1):1-14. doi: JME-16-0054 [pii].

35. Schneeberger M, Everard A, Gomez-Valades AG, et al. Akkermansia muciniphila inversely correlates with the onset of inflammation, altered adipose tissue metabolism and metabolic disorders during obesity in mice. Sci Rep. 2015;5:16643. doi: 10.1038/srep16643 [doi].

36. Everard A, Belzer C, Geurts L, et al. Cross-talk between akkermansia muciniphila and intestinal epithelium controls diet-induced obesity. Proc Natl Acad Sci U S A. 2013;110(22):9066-9071. doi: 10.1073/pnas.1219451110 [doi].

37. Cani PD, de Vos WM. Next-generation beneficial microbes: The case of akkermansia muciniphila. Front Microbiol. 2017;8:1765. doi: 10.3389/fmicb.2017.01765 [doi].

38. Goodrich JK, Waters JL, Poole AC, et al. Human genetics shape the gut microbiome. Cell. 2014;159(4):789-799. doi: 10.1016/j.cell.2014.09.053 [doi].

39. Zhang L, Bahl MI, Roager HM, et al. Environmental spread of microbes impacts the development of metabolic phenotypes in mice transplanted with microbial communities from humans. ISME J. 2017;11(3):676-690. doi: 10.1038/ ismej.2016.151 [doi].
40. Ridaura VK, Faith JJ, Rey FE, et al. Gut microbiota from twins discordant for obesity modulate metabolism in mice. Science. 2013;341(6150):1241214. doi: 10.1126/ science. 1241214 [doi].

41. Hwang I, Park YJ, Kim YR, et al. Alteration of gut microbiota by vancomycin and bacitracin improves insulin resistance via glucagon-like peptide 1 in diet-induced obesity. FASEB J. 2015;29(6):2397-2411.

42. Parseus A, Sommer N, Sommer F, et al. Microbiotainduced obesity requires farnesoid X receptor. Gut. 2016.

43. Cox LM, Yamanishi S, Sohn J, et al. Altering the intestinal microbiota during a critical developmental window has lasting metabolic consequences. Cell. 2014;158(4):705-721.

44. Cho I, Yamanishi S, Cox L, et al. Antibiotics in early life alter the murine colonic microbiome and adiposity. Nature. 2012;488(7413):621-626.

45. Nobel YR, Cox LM, Kirigin FF, et al. Metabolic and metagenomic outcomes from early-life pulsed antibiotic treatment. Nat Commun. 2015;6:7486.

46. Ussar S, Griffin NW, Bezy O, et al. Interactions between gut microbiota, host genetics and diet modulate the predisposition to obesity and metabolic syndrome. Cell Metab. 2015;22(3):516-530.

47. Turnbaugh PJ, Ley RE, Mahowald MA, Magrini V, Mardis ER, Gordon JI. An obesity-associated gut microbiome with increased capacity for energy harvest. Nature. 2006;444(7122):1027-1031. doi: nature05414 [pii].

48. Sonnenburg JL, Backhed F. Diet-microbiota interactions as moderators of human metabolism. Nature. 2016;535(7610):56-64. doi: 10.1038/nature18846 [doi].

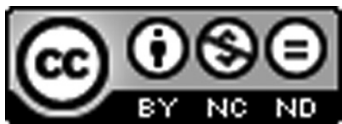

This work is licensed under a Creative Commons Attribution-Non Commercial-No Derivatives 4.0 International License. To view a copy of this license, visit http://creativecommons.org/licenses/by-nc-nd/4.0/ 